\title{
ARTICLE
}

\section{Spontaneous consecutive esotropia}

\author{
Nathalie Voide $\mathbb{I}^{1,2} \cdot$ Matthieu P. Robert ${ }^{1,3}$
}

Received: 11 November 2017 / Revised: 4 February 2018 / Accepted: 7 February 2018 / Published online: 2 March 2018

(c) The Royal College of Ophthalmologists 2018

\begin{abstract}
Purpose Although less frequent than consecutive exotropia, consecutive esotropia is a well-known type of strabismus when it follows the surgical correction of an exotropia. Spontaneous conversion from initial constant, large-angle exotropia beyond the age of 3 months to esotropia or orthophoria, however, is not common. We describe a series of infants who presented a spontaneous evolution from a large-angle infantile exotropia to either an orthophoria or a spontaneously consecutive esotropia. Methods Cases of infants examined in the pediatric neuro-ophthalmology clinic of a tertiary ophthalmology department between 2009 and 2015, and having presented an early large-angle exotropia that spontaneously converted into an esotropia or orthophoria-i.e., without any previous surgery or botulinum toxin injection—were studied.

Results Ten cases (6 M:4 F) were followed up. Median age at first exotropia assessment was 3.88 months $(\mathrm{SD}=6.35)$. Median age at spontaneous conversion to esotropia or orthophoria was 7.23 months $(\mathrm{SD}=14.73)$. Six patients suffered from severe neurologic or metabolic diseases, three had neonatal respiratory distress syndrome, and one was healthy.

Conclusion Spontaneous conversion from initial large-angle exotropia to esotropia or orthophoria can be encountered. The cerebral maturation of visual structures probably accounts for this uncommon strabismus sequence.
\end{abstract}

\section{Introduction}

Consecutive exotropia is currently defined as a transition from esotropia to exotropia secondary to a strabismus surgery or a botulinum toxin injection, with a possible gap of variable duration. The nosography has evolved: although "consecutive exotropia" was first used to designate an exotropia spontaneously following an initial esotropia [1], it is now almost exclusively used for postsurgical transitions.

The lack of normal retinal correspondence or the absence of fusion potential is usually associated with an esotropia in neurologically healthy children-the spontaneous conversions from eso- to exotropia being attributed to aging [2, 3]. The possibility of spontaneous transitions from exo- to

Nathalie Voide

nathalie.voide@fa2.ch

1 AP-HP, Department of Ophthalmology, Hôpital Universitaire Necker-Enfants malades, 149, rue de Sèvres, Paris 75015, France

2 Department of Ophthalmology, Jules Gonin Eye Hospital, Fondation Asile des Aveugles, University of Lausanne, 15, avenue de France, Lausanne 1004, France

3 Cognition and Action Group (COGNAC G), UMR 8257 CNRSIRBA-Université Paris Descartes, Sorbonne Paris Cité,

Paris, France esotropia in children without any previous strabismus surgery seems paradoxical.

We present a series of infants initially evaluated for an exotropia and having spontaneously evolved to esotropia or orthophoria.

\section{Methods}

The notes of the patients evaluated in the pediatric neuroophthalmology clinic of Necker-enfants malades hospital, Paris, between November 2009 and March 2015, who initially presented with a large-angle exotropia and spontaneously evolved to an esotropia or orthophoria, without any previous oculomotor surgery or botulinum toxin injection, were retrospectively collected. Sex, age, amenorrhea weeks at birth, age at exotropia onset, delay between exotropia onset, spontaneous conversion to esotropia or orthophoria, and cycloplegic refraction (using $0.3 \%$ atropine drops for 7 days and Retinomax autorefractor results) were studied. Owing to the young age of the infants, the initial orthoptic eye alignment evaluation was done by two experienced orthoptists and one ophthalmologist, using objects as fixation targets for attempted unilateral cover test but also always Hirschberg test in awake infants, considering the fact that $\kappa$-angle in infants is normally $8^{\circ}$ to 
$10^{\circ}$, so that decentered corneal reflections less than midway from the pupillary center do not indicate exotropia [4].

\section{Results}

Ten infants-six boys and four girls-were included. Results are presented in Table 1. Exotropia was constant and larger than 30 prism diopters in all cases. Median age at exotropia diagnosis was 3.88 months ( $\mathrm{SD}=6.35)$. Refraction showed no case of abnormal ametropia, no significant change of refraction was highlighted during the follow-up, and there was no case of amblyopia. Six children developed sudden large-angle esotropia at a median age of 19.43 months $(\mathrm{SD}=16.1)$ and four children developed orthophoria, all the latter with signs of normal stereoacuity (assessed by Lang and/or Wirt tests), at a median age of 5.58 months $(\mathrm{SD}=1.89)$. Considering that we are evaluating an average of about 30 new cases of early exotropia each year in our unit, the incidence of spontaneous conversion to esotropia or orthophoria was around $6 \%$.

\section{Discussion}

A series of 10 cases of spontaneous consecutive esotropia or orthophoria following early-onset large-angle exotropia is reported. The six children with consecutive esotropia were affected with a constitutive neurological condition, their general condition improved over time, and they never developed signs of normal retinal correspondence. On the other hand, the four infants having converted to orthophoria either had suffered from neonatal distress syndrome (three cases) or were healthy (one case), they showed signs of stereopsis, and they converted earlier than the six others. In infants with preexisting metabolic disorders, the introduction of enzymatic treatment seemed to favor conversion into esotropia.

Infantile exotropia is a rare condition. It is most often associated with cortical visual impairment in the context of term injury, more rarely with leuco-encephalopathies, either in the context of fixed periventricular leukomalacia in preterm babies or in association with an evolving-often metabolic_-disorder. The cases reported here constituted a typical population of infantile exotropia. As a rule, affected patients remain divergent [5]. The authors were able to find only one reported case of spontaneous consecutive esotropia, published in 1964; it occurred at the age of 3 years and 8 months in a healthy child [6]. "Dyskinetic strabismus" is a term coined by Buckley et al.[7] to designate a momentary fluctuation between exotropia and esotropia, typically unrelated to attention or accommodative effort, and encountered in patients with severe cerebral palsy, often with the absence of binocular fusion and severe gaze palsies
[8]. Although there may be links between the mechanisms of such angle instability and the present cases of consecutive esotropia, both conditions appear to be clinically distinct.

The relations between the development of eye alignment, convergence, and sensory binocularity are a matter of controversy. Difficulties partly result from the limitations in the examination of young infants. There is no consensus regarding the nature of ocular alignment in normal newborns: according to a first set of studies, most newborns (up to $72 \%$ at age 1 month) are exotropic $[9,10]$, whereas the other ones are orthotropic; according to others, the proportions are reverted (73\% of esotropia at age 1 month) [4, $11,12]$. In all studies, however, orthophoria is achieved in the majority of infants at age 3 months; in most reported cases of ocular exodeviations in normal newborns, the angles of deviation are small. Explanations for such differences between large series with robust methods probably lie partly on the fact that $\kappa$-angle is physiologically very large in newborn infants and decrease rapidly over the first few months of life $[13,14]$, and on differences resulting from techniques used for fixation targets [4]. In contrast to adults, it is known that infants under 4 months of age have not fully developed stereopsis; therefore, retinal disparity cannot yet activate fusional vergence eye movements, except for slow movements of limited amplitude [15]. As physiological cerebral immaturity can account for physiological neonatal eye exodeviation, delayed cerebral immaturity in infants affected by central diseases can probably explain most cases of true infantile exotropia.

A striking fact in this series is the strict correspondence between the nature of the associated condition and the evolution of the strabismus: all children affected with a constitutive neurological condition evolved to spontaneous consecutive esotropia at variable ages after the age of 6 months, whereas all infants with neonatal respiratory distress syndrome (whose central nervous system probably transiently suffered at birth) and the only healthy child of the series evolved to orthophoria with normal stereopsis before the age of 8 months. The switch to orthophoria and the possibility of establishing a normal retinal correspondence were probably driven by the early-enough presence of both good fusional vergence capacities and sensory visual performance. One may hypothesize that in case these conditions are lacking before the age of 8 months - i.e. before the end of the (or one of the) critical period(s) of visual development, which may be delayed in such cases compared with physiological situations-normal retinal correspondence and therefore orthophoria can never occur; however, fusional vergence can sometimes strengthen parallel to the neurological improvement and then naturally lead to spontaneous consecutive esotropia.

This series is a small one; the quantification of ocular deviations and $\kappa$-angles in small infants is always difficult. Although these biases were limited by the very large nature 


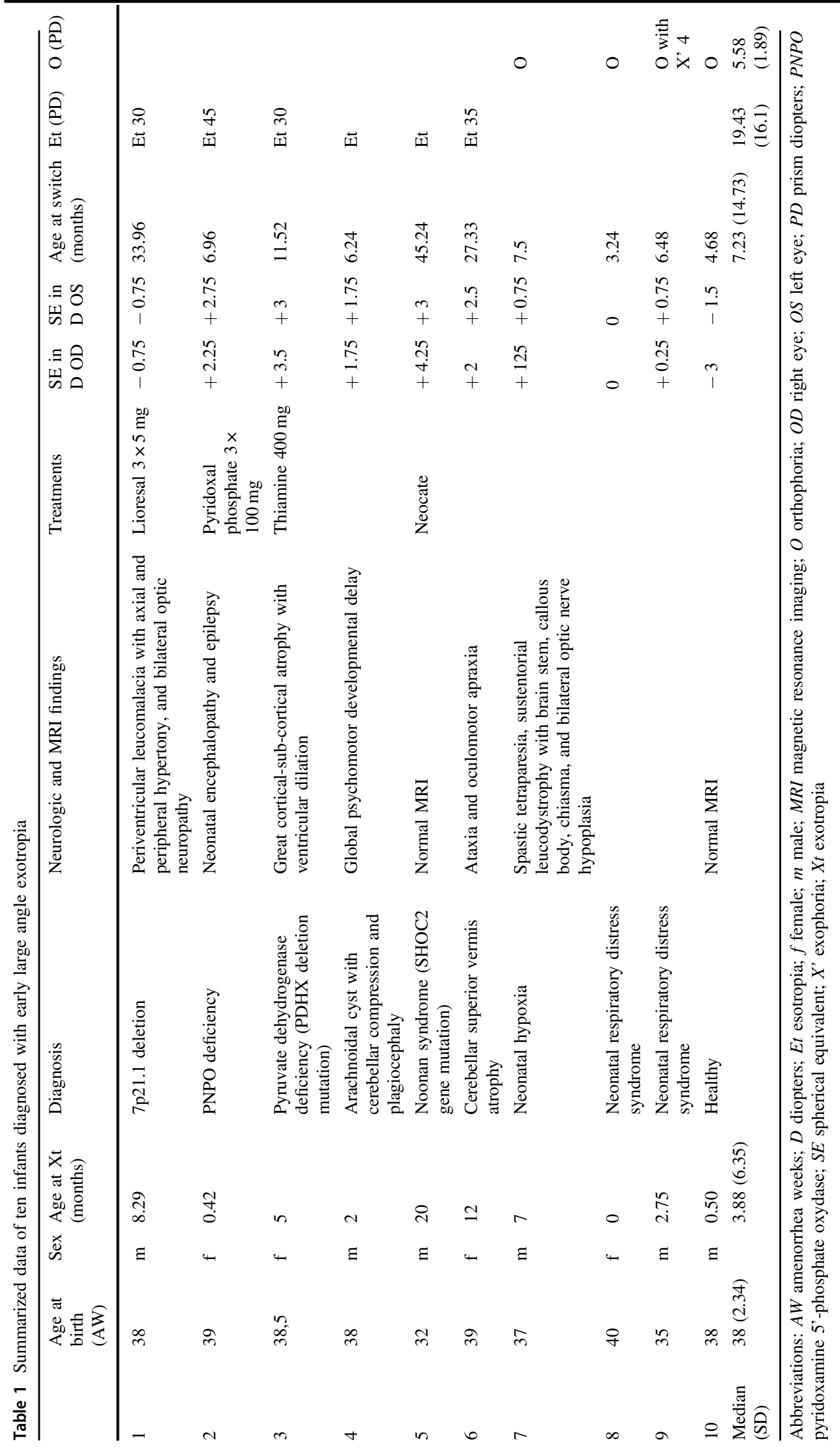


of the exodeviation angles in these children, further larger prospective studies are needed to better define and understand this clinical entity.

Spontaneous conversion from infantile large angle exotropia to orthophoria or esotropia do exist. It seems to occur in the context of early cerebral impairment followed by secondary improvement. In situations where a neurological improvement occurs before the age 8 months, the establishment of normal retinal correspondence can be achieved, with a switch to orthophoria and the establishment of good stereopsis; in cases where the improvement occurs later, a switch to spontaneous consecutive esotropia with abnormal retinal correspondence is observed. Delayed cerebral maturation during the first months of life in neurologically impaired infants offers a likely pathophysiologic hypothesis to this condition.

\section{Summary}

\section{What was known before}

- Although less frequent than consecutive exotropia, consecutive esotropia is a well-known type of strabismus when it follows the surgical correction of an exotropia.

- Spontaneous conversion from initial constant, largeangle exotropia beyond the age of 3 months to esotropia or orthophoria, however, is not common.

- The aim of this retrospective study is to qualify this uncommon phenomenon and try to classify the natural evolution of this strabismus conversion depending on the cerebral maturity.

\section{What this study adds}

- Spontaneous conversion from initial large-angle exotropia to esotropia or orthophoria can be encountered.

- In children with central neurologic disease, early exotropia could be favored by cerebral immaturity (neuronal maturation delay).

- In normal children, the constant ocular alignment and the mature fusional vergence activated by retinal disparity are acquired at about 4 months of life.

- Children with less severe, acquired, central disease have a more early conversion from initial large-angle exotropia to orthophoria before 8 months of life and are able to develop fusional vergence and normal retinal correspondence.

- However, children with severe, constitutional central disease activate convergence mechanisms probably after the sensitive period of 6-8 months of life and convert to esotropia.

Acknowledgements We thank Charlotte Creux and Marie Saudubray who performed of the orthoptic evaluations, and also Professor Pascale de Lonlay, Dr Vassili Valayannopoulos, and Professor Desguerre, who followed and treated the children with metabolic disorders.

Financial disclosure There is no financial interest.

\section{Compliance with ethical standards}

Conflict of interest The authors declare that they have no conflict of interest.

\section{References}

1. Hugonnier R, Hugonnier S. Physiologie des mouvements oculaires. Strabismes. Hétérophories. Paralysies oculomotrices. 4th edition, Masson, Paris, 1981.

2. Senior JD, Chandna A, O'Connor AR. Spontaneous exotropia in childhood. Strabismus. 2009;17:33-6.

3. Weir CR, Cleary M, Dutton GN. Spontaneous exotropia in children with motor fusion. Br J Ophthalmol. 2001;85: 242-3.

4. Thorn F, Gwiazda J, Cruz AA, Bauer JA, Held R. The development of eye alignment, convergence, and sensory binocularity in young infants. Invest Ophthalmol Vis Sci. 1994;35:544-53.

5. Brodsky MC. Pediatric neuro-ophthalmology 307-315 (Springer, New-York, 2010).

6. Fitton MH, Jampolsky A. A case report of spontaneous consecutive esotropia. Am Orthopt J. 1964;14:144-6.

7. Buckley E, Seaber JH. Dyskinetic strabismus as a sign of cerebral pasly. Am J Ophthalmol. 1981;91:652-7.

8. Ghasia F, Brunstrom J, Grodon M, Tychsen L. Frequency and severity of visual sensory and motor deficits in children with cerebral palsy: gross motor function classification scale. Invest Ophthalmol Vis Sci. 2008;49:572-80.

9. Sondhi N, Archer SM, Helveston EM. Development of normal ocular alignment. J Pediatr Ophthalmol Strab. 1988;25: $210-1$.

10. Archer SM, Sondhi N, Helveston EM. Strabismus in infancy. Ophthalmology . 1989;96:133-7.

11. Nixon RB, Helveston EM, Miller K, Archer SM, Ellis FD. Incidence of strabismus in neonates. Am $\mathrm{J}$ Ophthalmol. 1985;100:798-801.

12. Horwood AM. Neonatal ocular misalignments reflect vergence development but rarely become esotropia. $\mathrm{Br} \mathrm{J}$ Ophthalmol. 2003;87:1146-50.

13. Slater AM, Findlay JM. Binocular fixation in the newborn baby. Exp Child Psychol. 1975;20:248-73.

14. Riddell PM, Hainline L, Abramov I. Calibration of the Hirschberg test in human infants. Invest Ophthalmol Vis Sci. 1994;35:538-43.

15. Horwood AM, Riddell PM. Can misalignments in typical infants be used as a model for infantile esotropia? Invest Ophthalmol Vis Sci. 2004;45:714-20. 